\title{
ANALYZING SPECTRAL CHARACTERISTICS OF SHADOW AREA FROM ADS-40 HIGH RADIOMETRIC RESOLUTION AERIAL IMAGES
}

\author{
Yi-Ta Hsieh ${ }^{\mathbf{a}}$, Shou-Tsung Wu ${ }^{\mathbf{b}}$, Chaur-Tzuhn Chen ${ }^{\mathbf{c}}$, and Jan-Chang Chen ${ }^{\mathbf{c}, *}$ \\ ${ }^{\text {a }}$ Department of Graduate Institute of Bioresources, National Pingtung University of Science and Technology, No.1, Shuehfu Rd., \\ Neipu, Pingtung 91201, Taiwan; E-Mails: blue90234570@ gmail.com \\ ${ }^{b}$ Department of Tourism Management, Shih Chien University, No.200 University Rd., Neimen, Kaohsiung 84550, Taiwan; E-Mail: \\ stwu@mail.kh.usc.edu.tw \\ c Department of Forestry, National Pingtung University of Science and Technology, No.1, Shuehfu Rd., Neipu, Pingtung 91201, \\ Taiwan; Chaur-Tzuhn Chen E-Mails: cct@gisfore.npust.edu.tw; Jan-Chang Chen E-Mails: zzzjohn@ gisfore.npust.edu.tw \\ * Author to whom correspondence should be addressed; E-Mail: zzzjohn@mail.npust.edu.tw
}

Commission VII, WG VII/4

KEY WORDS: Shadow, shadow spectra, ADS-40, high radiometric resolution

\begin{abstract}
:
The shadows in optical remote sensing images are regarded as image nuisances in numerous applications. The classification and interpretation of shadow area in a remote sensing image are a challenge, because of the reduction or total loss of spectral information in those areas. In recent years, airborne multispectral aerial image devices have been developed 12-bit or higher radiometric resolution data, including Leica ADS-40, Intergraph DMC. The increased radiometric resolution of digital imagery provides more radiometric details of potential use in classification or interpretation of land cover of shadow areas. Therefore, the objectives of this study are to analyze the spectral properties of the land cover in the shadow areas by ADS-40 high radiometric resolution aerial images, and to investigate the spectral and vegetation index differences between the various shadow and non-shadow land covers. According to research findings of spectral analysis of ADS-40 image: (i) The DN values in shadow area are much lower than in nonshadow area; (ii) DN values received from shadowed areas that will also be affected by different land cover, and it shows the possibility of land cover property retrieval as in nonshadow area; (iii) The DN values received from shadowed regions decrease in the visible band from short to long wavelengths due to scattering; (iv) The shadow area NIR of vegetation category also shows a strong reflection; (v) Generally, vegetation indexes (NDVI) still have utility to classify the vegetation and non-vegetation in shadow area. The spectral data of high radiometric resolution images (ADS-40) is potential for the extract land cover information of shadow areas.
\end{abstract}

\section{INTRODUCTION}

During the image capturing process of very high resolution imagery, numerous influential factors hinder the quality of these images, such as the shadows caused from the different angle of the sun, terrain features, and surface object occlusion (Dare, 2005; Tsai, 2006). Furthermore, the shadows in remote sensing images are regarded as image nuisances in numerous applications, specifically, change detection and image classification (Dare, 2005; Zhou et al., 2009), frequently affecting the accuracy of analytical results (Wilson, 1997). Especially in the mountainous environment, shadows frequently occur in terrain areas with steep slopes (Giles, 2001; Dare, 2005). As a result, the accuracy of land cover/ use mapping procedure over steep mountainous terrain is often low (Dorren et al., 2003; Shahtahmassebi et al., 2013). In Taiwan, the landscape is often characterized as alpine terrain, thus using very high resolution images for land cover/ use mapping will be severely affected by the shadow problem.

The classification and interpretation of the shaded area in remote sensing image is a challenge, because of the reduction or total loss of spectral information in those areas (Dare, 2005; Yuan, 2008; Zhou et al., 2009), hence the importance of understanding the spectral characteristics of such areas, for fundamental analysis.
In theory, in an optical image, shadows are formed by obstructing direct light. Under these conditions, target objects in a shadow area are irradiated by the scattered light and reflected light from the surrounding environment (Chakraborti, 2007; Makarau et al., 2011; Adeline et al., 2013; Wu et al., 2014). Conversely, target objects in a non-shadow area not only receive scattered and reflected light, but also direct light. The scattered light in shadow areas is influenced by Rayleigh scattering, Mie scattering, and differential atmospheric scattering, with Rayleigh scattering being the most influential ( $\mathrm{Gu}$ and Robles-Kelly, 2014). In the range of visible light and near infrared light, the intensity of Rayleigh scattering is inversely related to the fourth power of wavelength. Thus, the intensity of the scattered light drastically decreases with increased wavelengths (Jensen, 2005). In Adeline et al. (2013)'s paper simulating the spectral characteristics of asphalt within a shadow area, and the results indicated sensor radiance received from shadowed regions decreased from short to long wavelengths due to scattering. However, in the past, conventional telemetry data using 8-bit radiometric resolution in shadow area, could only record 10-20 on the grayscale range, even less with a 5. As previously noted, the scattering effect is more difficult to detect from a 8-bit data. Only analog data can be used in a way that relates to the support of spectral characteristics in empirical data. 
In recent years, airborne multispectral aerial image devices have produced high radiometric resolution data (12-bit or higher), providing more radiometric details for potential use in classification or interpretation of land cover of shadow areas (Wu et al. 2014). High radiometric resolution data offers great possibilities for the observation of the spectral characteristics of shadow area and solving the shadow problem .

However, few studies focused on how high radiometric resolution data was dealt with the shadow problem $(\mathrm{Wu}$ et al. 2014), and worked on classifying or interpreting the land cover of shadow areas in remote sensing images (Zhou et al. 2009; Wu et al. 2014). Even, fewer ones are analyzing the spectral properties in the shadow areas. Therefore, the objectives of this study is to analyze the spectral properties of the land cover in the shadow areas by ADS-40 high radiometric resolution aerial images, and to investigate the spectral and vegetation index differences between the various shadow and non-shadow land covers. To provide telemetry data to support the theory of spectral characteristics of the shadow area.

\section{STUDY AREA AND MATERIALS}

\subsection{Study area}

The research site is located at the Da-Chia stream working cycle, Central Taiwan. The landscape of the research site is characterized as alpine terrain. Its elevation ranges between 743-3,882 m, and shows significant topographical changes. DaChia stream working cycle is under the jurisdiction of Dongshi Forest District Office of the Forestry Bureau, total 85 compartments contained. The area is located in upstream of DaChia stream and Peikang stream, between Syushan and Central Mountain. With large elevation variations, it results in three kinds of climate type: warm, temperate, frigid zones. The vegetation distribution of broadleaf forest type is at 2,000 m. At $2,000-2,800 \mathrm{~m}$ is the conifer-hardwood forest type; vegetation above $2,800 \mathrm{~m}$ includes the conifer trees and fargesia. The largest species of conifer forest types is Pinus taiwanensis Hyata (Liu and Su, 1978). The site is composed mostly of natural forest (Table 1). The land cover for this site includes non-vegetation (e.g., buildings, roads, exposed riverbeds, and exposed orchards), vegetation (e.g., natural forests, plantation, grasslands, and orchards), and water (e.g., streams) as shown in Figure 1.

\begin{tabular}{lr}
\hline Land use & Area (ha, \%) \\
\hline Natural forest & $325000,68.6$ \\
Plantation & $11600,24.5$ \\
Grassland & $2200,4.6$ \\
Dry land, orchard, others & $1100,2.3$ \\
\hline
\end{tabular}

Table 1. Composition of land cover of the study area

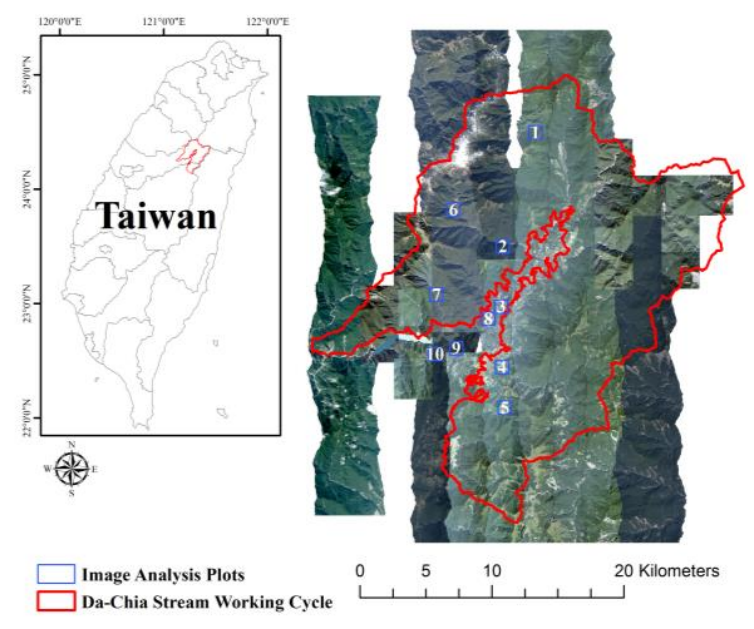

Figure 1. The location of the study area (the numbers in map are plot numbers).

\subsection{Materials}

Two kinds of camera were used for this study: Leica ADS40 and Z/I DMC. The capturing of ADS-40 and DMC images were conducted by the Aerial Survey Office, Forestry Bureau of Taiwan (Figure 1). Digital Aerial Images were captured between 2007/10/27 and 2009/01/02 (most in 2008/09).

The ADS-40 images underwent a series of image processing procedures to produce Level 2 multispectral ortho-image product, in which the Leica Photogrammetry Suite (LPS), Orima, and OrthoVista processing software were used for aerotriangulation and orthorectification procedures, and the Leica Inertial Position and Attitude System (IPAS) and the Waypoint GrafNav/GrafNet software were used for inertial measurement unit and global positioning system (GPS/IMU) processing (Yeh et al., 2010). The spatial resolution of the ADS-40 ortho-images was $25 \mathrm{~cm}$, providing detailed surface properties. The ADS-40 image comprised four multispectral bands (namely, red $(\mathrm{R})=$ wavelength $610-660 \mathrm{~nm}$; green $(\mathrm{G})=$ wavelength $535-585 \mathrm{~nm}$; blue $(B)=$ wavelength $430-490 \mathrm{~nm}$; and near-infrared $(\mathrm{NIR})=$ wavelength $835-885 \mathrm{~nm}$ ). In addition the images presented 14-bit radiometric resolution and excellent signal to noise ratios. The multispectral band data can be recorded up to a 13-bit (digital number (DN) range: 0-8,191) grayscale range, and the panchromatic band can be recorded up to a 14-bit (DN range: 0-16,383) grayscale range (Pateraki and Baltsavias, 2003). The multispectral band was analyzed in this study. The images were stored in a 16-bit format, but retained their original radiometric resolutions (multispectral band $=13$ bits).

The DMC images also underwent a series of image processing procedures to produce multispectral ortho-image productions, in which the ISAT (ImageStation Automatic Triangulation), OrthoPro and OrthoVista processing software were used for aerotriangulation, orthorectification, image equalizer and image mosic procedures, and the Applanix POSPac (Position \& Orientation System) software were used for inertial measurement unit and global positioning system (GPS/IMU) processing (Yeh et al., 2010). The spatial resolution of the DMC ortho-images was $25 \mathrm{~cm}$. The DMC image comprised four multispectral bands (i.e., red $(\mathrm{R})=$ wavelength $590-675 \mathrm{~nm}$; green $(\mathrm{G})=$ wavelength $500-650 \mathrm{~nm}$; blue $(\mathrm{B})=$ wavelength 
400-580 nm; and near-infrared (NIR) = wavelength $675-850$ $\mathrm{nm})$. The multispectral band was analyzed in this study, and the images were stored in a 16-bit format, but retained their original radiometric resolutions (multispectral band $=12$ bits).

\section{METHODS}

\subsection{Training data selection}

In this study, we set 10 image analysis plots (Figure 1), and a spectral analysis was performed for each plot. Each plot has two periods of images, one is a First period image, and the other is the Second period image (different captured time from the same location in a second period)(Table 2).

\begin{tabular}{|llllll|}
\hline & \multicolumn{2}{l}{ First period images } & & \multicolumn{2}{c|}{ Second period images } \\
\cline { 2 - 3 } \cline { 5 - 6 } & Sensor & Date & & Sensor & Date \\
\hline Plot 1 & ADS-40 & $2008 / 09 / 21 / 10: 42$ & & DMC & $2008 / 08 / 03 / 11: 01$ \\
Plot 2 & ADS-40 & $2009 / 01 / 02 / 12: 22$ & & ADS-40 & $2008 / 09 / 21 / 09: 58$ \\
Plot 3 & ADS-40 & $2008 / 09 / 21 / 09: 58$ & & ADS-40 & $2008 / 09 / 17 / 12: 23$ \\
Plot 4 & ADS-40 & $2008 / 12 / 27 / / 11: 20$ & & ADS-40 & $2008 / 09 / 21 / 10: 20$ \\
Plot 5 & ADS-40 & $2008 / 12 / 27 / 11: 20$ & & ADS-40 & $2008 / 09 / 21 / 10: 20$ \\
Plot 6 & ADS-40 & $2009 / 01 / 02 / 12: 05$ & & DMC & $2008 / 08 / 26 / 11: 07$ \\
Plot 7 & ADS-40 & $2009 / 01 / 02 / 12: 05$ & & ADS-40 & $2008 / 09 / 19 / 11: 29$ \\
Plot 8 & ADS-40 & $2008 / 09 / 21 / 09: 50$ & & ADS-40 & $2008 / 09 / 17 / 12: 31$ \\
Plot 9 & ADS-40 & $2008 / 09 / 21 / 10: 05$ & & ADS-40 & $2008 / 09 / 19 / 11: 29$ \\
Plot 10 & ADS-40 & $2008 / 09 / 21 / 10: 05$ & & ADS-40 & $2008 / 09 / 19 / 11: 29$ \\
\hline
\end{tabular}

Table 2. Data use of 10 image analysis plots

The DN values of the surface objects in the shadow areas were statistically analyzed and simultaneously compared with values in the nonshadow areas. To analyze the spectral values of the various shadow surface objects, we overlapped the first period image and second period image to select the training data of shadow areas from the first period image, and digitally established the polygons for nonvegetation, vegetation, and water bodies in these areas. Because the land cover in the shadow region could not be interpreted, the training sets of the three land covers were interpreted by using a second period image as the reference image. Therefore, the shadow areas of first period image and nonshadow areas of the second period image were used for the selection range.

We also calculated the Normalized Difference Vegetation Index (NDVI) image as the auxiliary data to help the interpretation of the land cover of the shadow area, because the NDVI could show some land cover information in the shadow area (Equation (1)).

$$
N D V I=\frac{N I R-R E D}{N I R+R E D}
$$

In addition to selecting the training sets for the shadow regions, the training sets for non-shadow areas in the first period image were also tabbed. Next, the training sets range was used to extract the pixel values for the various bands in the first period image.

\subsection{Spectral analysis}

This study conducted descriptive statistical analyses, exploratory data analysis, and investigated the multispectral data differences between the various shadow and non-shadow land covers. This study also investigated the vegetation index (NDVI) differences between the various shadow and nonshadow land covers.

\section{RESULTS AND DISCUSSION}

\subsection{Spectral characteristics of the shadow area}

The spectral properties are extracted from the bands of shadow and non-shadow areas for the various land cover types. The descriptive statistics of DN values of all plots are shown in Table 3 and Table 4. Figure 2 that the DN distribution for the differing bands of various surface objects presented significant differences between the shadow and non-shadow area.

The DN values with smaller value ranges in the shadow area are primarily distributed below 1,100 with smaller value ranges, whereas the DNs in the non-shadow area are mostly distributed above 400 with larger value ranges (Table 3 ). Lower DN values were observed in the shadow areas in ADS-40 image (Table 3). In an optical image, shadows are formed by obstructing direct light. The lower DN values in the shadow areas cause partial or total loss of radiometric information in the affected areas (Dare, 2005; Yuan, 2008; Zhou et al., 2009), the loss of radiometric information is the absence of direct light (Adeline et al., 2013; Wu et al., 2014).

\begin{tabular}{|lrrllll|}
\hline & \multicolumn{2}{c}{ Vegetation } & \multicolumn{2}{c}{ Non-vegetation } & \multicolumn{2}{c|}{ Water body } \\
& Mean & SD & Mean & SD & Mean & SD \\
Red & 229.46 & 69.31 & 312.85 & 93.96 & 377.75 & 87.73 \\
Green & 433.65 & 112.82 & 521.41 & 121.31 & 629.20 & 135.27 \\
Blue & 709.21 & 104.60 & 829.62 & 109.79 & 934.07 & 135.19 \\
NIR & 597.65 & 312.46 & 397.53 & 259.54 & 341.67 & 220.98 \\
\hline
\end{tabular}

Table 3. Summary of descriptive statistics of DN values of shadow area

\begin{tabular}{|lcccccc|}
\hline & \multicolumn{2}{c}{ Vegetation } & \multicolumn{2}{c}{ Non-vegetation } & \multicolumn{2}{c|}{ Water body } \\
& Mean & SD & Mean & SD & Mean & SD \\
\hline Red & 697.90 & 188.34 & 2061.13 & 1028.21 & 1658.22 & 629.34 \\
Green & 1124.51 & 267.43 & 2284.64 & 1153.26 & 2083.23 & 813.78 \\
Blue & 1046.91 & 133.23 & 2030.27 & 862.81 & 1901.10 & 589.07 \\
NIR & 3198.56 & 849.49 & 1652.61 & 643.33 & 707.21 & 306.37 \\
\hline
\end{tabular}

Table 4. Summary of descriptive statistics of DN values of nonshadow area

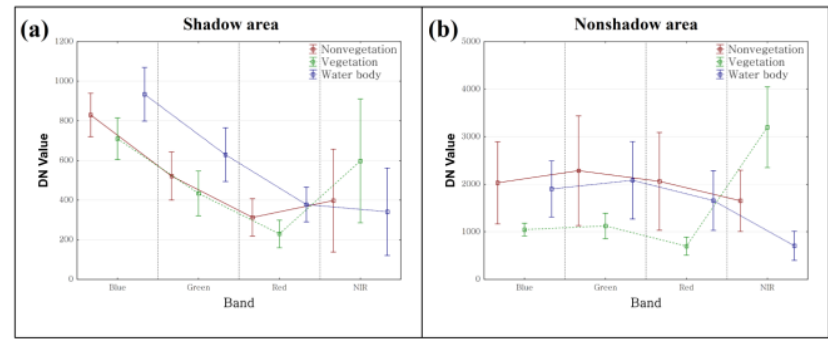

Figure 2. The multispectral image for training spectra (digital number value) in (a) shadow areas and (b) nonshadow areas. Note: Center squares indicate mean, and the whisker one standard deviation.

In addition, the mean $\mathrm{DN}$ values of all plots for the $\mathrm{R}, \mathrm{G}$, and $\mathrm{B}$ bands in the non-shadow area are non-vegetation>water bodies $>$ vegetation, whereas those in the shadow area are water bodies>non-vegetation>vegetation (Table 3 and Table 4) (Figure 2). Regardless of shadow or non-shadow, the DN values for vegetation in the NIR band are significantly highest than 
water bodies and non-vegetation, indicating that NIR effectively reflects vegetation conditions (Table 3 and Table 4) (Figure 2). We note that different land covers have different spectral curves (Figure 2). DN values received from shadowed areas that were also affected by different land covers.

The waveforms for the shadow and non-shadow areas were noticeably different. The waveform for the shadow areas did not compress the value ranges in a proportion similar to the nonshadow areas, presenting significant spectral differences. Especially, the DN values for the shadow area, decreased in correspondence with the lengthening of the visible bands (i.e., R, G, and B). We actually employed the ADS-40 high radiometric resolution data for analysis of the spectral characteristics of shadow, and we got similar results as Adeline et al. (2013), which indicated that the spectrum of the shadow areas was significantly influenced by Rayleigh scattering. Furthermore, according to the spectral distributions of various land covers, non-vegetation and water bodies displayed typical spectral curves in the non-shadow areas (Figure 2). Because of Rayleigh scattering effect, the DN values of shadow areas for the non-vegetation and water bodies decreased with the lengthening of the wavelengths (Figure 2).

The spectral distributions of various land covers, nonvegetation and water bodies displayed typical spectral curves in the non-shadow area (Figure 2b). However, non-vegetation and water bodies in the shadow area displayed different spectral curves, and from $\mathrm{B}, \mathrm{G}$ and presented a drastic decrease to $\mathrm{R}$ (Figure 2a). Vegetation elements in the non-shadow area displayed a typical spectral curve, $\mathrm{R}$ and $\mathrm{B}$ presented absorption valley, $G$ presented a minor reflection peak and NIR presented a major reflection peak is due to the biophysical properties of the plant (Jensen, 2005) (Figure 2b). However, vegetation elements in the shadow areas displayed a different spectral curve. Due to the effects of Rayleigh scattering, B and G presented a drastic decrease to R, and NIR show a strong reflection (Figure 2a). We can note that different land covers have different spectral curves. Visible and near-infrared reflection of shadows are significantly affected by the surface type (Leblon et al., 1996). DN values received from shadowed areas that will be also affected by different land cover as from nonshadow area.

\subsection{Characteristics of vegetation index in shadow area}

This study also analyzed the characteristics of vegetation index in the shade area and non-shadow area. The vegetation indexes (NDVI) is calculated from the bands of the shadow and nonshadow area for the various land cover types.

We compare NDVI mean values between shadow and nonshadow area, the results indicated that shadowing decline NDVI mean values from 0.64 to 0.38 in the vegetation category, increase NDVI mean values from -0.10 to 0.03 in the nonvegetation category, and also increase NDVI mean values from -0.41 to -0.13 in the water body category (Table 5) (Figure 3 ). Those results are indicated that shadowing decline NDVI mean values in vegetation category, but increase NDVI mean values in non-vegetation and water body category. Compared to other works, Zhou et al. (2009) tried to classify between vegetation and artificial building in the shadow areas, where the shadow area using NDVI $=0.1$ as the classification threshold, while in the non-shadow area distinguish between vegetation and artificial building is used NDVI $=0.05$ as the classification threshold, and results showed shadow area of vegetation classification threshold is higher than non-shadow situation.
This phenomenon was also reported in Hsieh et al. (2011), they are trying to classify between vegetation and landslide in the shadow areas, and the results also showed the shadow area of vegetation classification threshold is higher than non-shadow situation. NDVI is conducted from R and NIR. However, the reasons for vegetation indexes differences between shadow area and non-shadow area are mainly affected by the reactions from $\mathrm{R}$ and NIR of this study. Generally the vegetation index of shadow area is still able to classify the vegetation and nonvegetation.

\begin{tabular}{|lcccccc|}
\hline & \multicolumn{2}{c}{ Vegetation } & \multicolumn{2}{c|}{ Nonvegetation } & \multicolumn{2}{c|}{ Water body } \\
& Mea & SD & Mean & SD & Mean & SD \\
\hline $\begin{array}{l}\text { Shadow area } \\
\text { NDVI }\end{array}$ & 0.38 & 0.20 & 0.03 & 0.27 & -0.13 & 0.27 \\
$\begin{array}{l}\text { Nonshadow } \\
\text { area NDVI }\end{array}$ & 0.64 & 0.07 & -0.10 & 0.17 & -0.41 & 0.17 \\
\hline
\end{tabular}

Table 5. Descriptive statistics of vegetation indexes of the shadow and non-shadow areas for the various land cover types

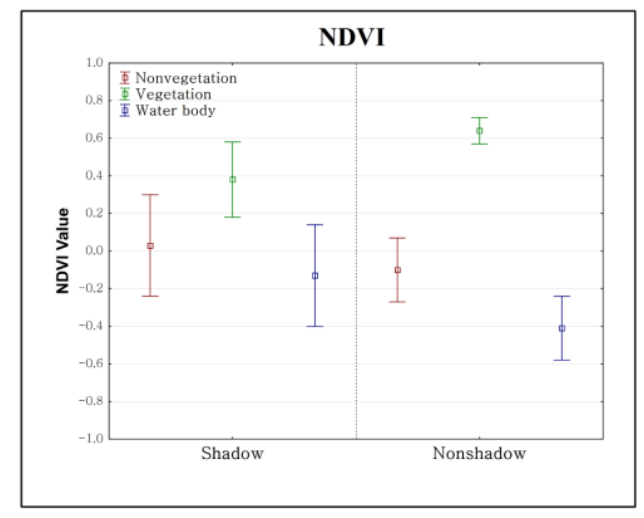

Figure 3. The vegetation index (NDVI) in shadow and nonshadow area; Note: Center squares indicate mean, and the whisker one standard deviation.

\section{CONCLUSIONS}

In order to evaluate how shadow processing methods, high radiometric resolution images can help eliminate the shadow problem, and improve land use/ cover mapping techniques. This study made use of high radiometric resolution digital aerial images to investigate the shadow issue using remote sensing techniques. Because the 13-bit multispectral remote-sensing data, spectral properties of the shadow areas were clearly observed and subsequently, because the shadow areas obstructed direct sunlight, the opportunity to observe scattered light was increased. The spectral properties of the shadow areas varied from those of the non-shadow areas. Therefore, these areas were separately processed during digital image analysis (e.g. image classification). According to the research findings of the spectral analysis of ADS-40 images, the following summary can be generalized about the spectral characteristics of the shadow area:

(1) The DN values in shadow area are much lower than in non-shadow area.

(2) $\mathrm{DN}$ values received from shadowed areas that will be also affected by different land cover, and showing the possibility of land cover property retrieval as in nonshadow area. 
(3) The DN values received from shadowed regions decreases in the visible light from short to long wavelengths due to scattering.

(4) The shadow area NIR of vegetation category also shows a strong reflection.

(5) Vegetation index (NDVI) has the function to classify the vegetation and non-vegetation in the shadow area.

High radiometric resolution data can resolve the spectral characteristics of the shadow area. However, the study did not consider the effect of cast-shadow (umbra, penumbra) and selfshadow for the differences in the spectrum around the object, nor suggestions for further depth analysis. Future work may conduct shadow compensation by spectral characteristics of shadow, such as light scattering theory-based shadow compensation mode. Vegetation indexes have the function to classify the vegetation and non-vegetation in shadow area, which can estimate the ratio of green cover of shadow area and detect the landslide of shadow area, to improve mapping accuracy.

\section{ACKNOWLEDGEMENTS}

The authors would like to thank the Aerial Survey Office, Forestry Bureau of Taiwan, for providing the ADS-40, DMC multispectral images for this study.

\section{REFERENCES}

Adeline, K. R. M., M. Chen, X. Briottet, S. K. Pang, and N. Paparoditis, 2013. Shadow detection in very high spatial resolution aerial images: A comparative study. ISPRS Journal of Photogrammetry and Remote Sensing, 80, pp.21-38.

Chakraborti, S., 2007. Verification of the Rayleigh scattering cross section. American Journal Physiology, 75(9), pp. 824-826.

Dare, P.M., 2005. Shadow analysis in high-resolution satellite imagery of urban areas. Photogrammetric Engineering \& Remote Sensing 71(2), pp. 169-177.

Dorren, L. K. A, B. Maier, and A. C. Seijmonsbergena, 2003. Improved Landsat-based forest mapping in steep mountainous terrain using object-based classification. Forest Ecology and Management, 183(1-3), pp. 31-46.

Doxaran, D., J. M. Froidefond, and P. Castaing, 2002. A reflectance band ratio used to estimate suspended matter concentrations in sediment-dominated coastal waters. International Journal of Remote Sensing, 23(23), pp. 50795085 .

Giles, P., 2001. Remote sensing and cast shadows in mountainous terrain. Photogrammetric Engineering \& Remote Sensing, 67(7), pp. 833-839.

Gu L., Robles-Kelly A., 2014. Shadow modelling based upon Rayleigh scattering and Mie theory. Pattern Recognition Letters, 43, pp. 89-97.

Hsieh, Y. T., S. T. Wu, C. S. Liao, Y. G. Yui, J. C. Chen, and Y. L. Chung, 2011. Automatic extraction of shadow and nonshadow landslide area from ADS-40 image by stratified classification methodology.p In: Proceedings of the 2011 IEEE International Geoscience and Remote Sensing Symposium (IGARSS). July 24-29, 2011. Vancouver, 2011.
Jensen, J.R., 2005. Introductory Digital Image Processing: A Remote Sensing Perspective, 3rd Edition, Upper Saddle River: Prentice-Hall, $526 \mathrm{p}$.

Leblon, B., b. Lisa, and H. Granberg, 1996. Effect of shadowing type on ground-measured visible and near-infrared shadow reflectances. Remote Sensing and Environment, 58, pp. 322328.

Liu, T. S., and H. J. Su, 1978. Studies on the community composition and the environmental factors of the natural foredts of Taiwan Red Pine in the upper Ta-chia valley.(in Chinese). Journal of the Experimental Forest of National Taiwan University, 121, pp.: 207-239.

Makarau, A., R. Richter, R. Muller, and P. Reinartz, 2011. Adaptive shadow detection using a blackbody radiator model. IEEE Trans. Geosci. Remote Sensing, 49, pp. 2049-2059.

Pateraki, M., and Baltsavias, E., 2003. Analysis and Performance of the Adaptive Multi-image matching Algorithm for Airborne Digital Sensor ADS40. In ASPRS Annual Conference, 5-9 May, Anchorage, AK, USA, (on CD-ROM).

Shahtahmassebi, A., N. Yang, K. Wang, N. Moore, and Z. Shen, 2013. Review of shadow detection and de-shadowing methods in remote sensing. Chinese Geographical Science, 23(4), pp. 403-420.

Tsai, V. U. D., 2006. A comparative study on shadow compensation of color aerial images in invariant color models. IEEE Transactions on Geoscience and Remote Sensing, 44(6), pp. 1661-1671.

Wilson, P.A., 1997. Rule-based classification of water in Landsat MSS images using the variance filter. Photogrammmetric Engineering \& Remote Sensing, 63(5), pp. 485-491.

Wu, S. T., Y. T. Hsieh, C. T. Chen, and J. C. Chen, 2014. A Comparison of 4 Shadow Compensation Techniques for Land Cover Classification of Shaded Areas from High Radiometric Resolution Aerial Images. Canadian Journal of Remote Sensing, 40(4), pp. 315-326.

Yeh, K. S., Li, M. Y., and Wang, Y. H., 2010. Applying digital aerial images in natural hazards mitigation in Taiwan. (in chinese). Journal of Photogrammetry and Remote Sensing, 15(1), pp. 123-140.

Yuan, F., 2008. Land-cover change and environmental impact analysis in the Greater Mankato area of Minnesota using remote sensing and GIS modeling. International Journal of Remote Sensing, 29(4), pp. 1169-1184.

Zhou, W., G. Huang, A. Troy, and M. L. Cadenasso, 2009. Object-based land cover classification of shaded areas in high spatial resolution imagery of urban areas: A comparison study. Remote Sensing of Environment, 113, pp. 1769-1777. 